\title{
Optimization of FES-assisted rising motion in individuals with paraplegia
}

\author{
Jovic Jovana ${ }^{*}$ Azevedo Coste Christine ${ }^{*}$ Fraisse Philippe ${ }^{*}$ Bonnet Vincent ${ }^{\ddagger}$ Fattal Charles $* \dagger$ \\ (*)DEMAR LIRMM/INRIA Montpellier, France \\ (†)LABLAB Rome, Italy \\ (*))PROPARA Centre Montpellier, France
}

\section{E-mail:jovic@lirmm.fr, azevedo@lirmm.fr,fraisse@lirmm.fr,v.bonnet@uniroma4.it, c.fattal@propara.languedoc-mutalite.fr}

\begin{abstract}
The objective of this paper is to investigate what would be the optimal strategy for voluntary trunk movement, which would minimize hip, knee and ankle torques demanding as well minimal upper limb participation during Functional Electrical Stimulation (FES)-assisted sit to stand motion in person suffering from Spinal cord injury. Our results suggest that paraplegic patients should bend their body forward in order to use linear momentum of the trunk in sit off phase, i.e. they should generate the motion similar to the one of healthy subjects. Those results have been experimentally tested using a closed-loop controller for FES-assisted standing-up. The controller should automatically trigger leg stimulation in optimal moment with respect to the trunk motion in order to decrease arm participation during rising phase of the motion.
\end{abstract}

\section{Introduction}

Standing-up is one of the most common daily activities in healthy people. It is a mechanically demanding task [1] and requires active force provided by muscles of the lower limbs. Spinal cord injury patients have difficulties in standing up, due to their lower limbs paralysis. To compensate missing action of lower extremities, paraplegic patients use arm support to provide lifting and stabilizing forces during standing up. However, people practicing a fully arm supported standing-up risk later shoulder complications [11]. In addition to the arm support, standing up in paraplegic patients can be achieved by using FES. Previous work in our group had shown that orientation and acceleration of the healthy human trunk during rising motion have low intra and intervariability, so those signals could be good indicators of the evolution of sit to stand movement [2]. Also, comparing rising motion in healthy and paraplegic subjects it has been demonstrated that one main difference between them is the onset of leg movement in regards to trunk bending [2],[10]. Thus, the system for closed-loop FES assisted sit-to-stand transfer, based on the observation of trunk movement has been developed [3]-[4]. The method is based on the observation of trunk acceleration in the sagittal plane during rising motion and a detection algorithm which triggers a pre-programmed stimulation pattern. The detection algorithm is described in [4]. Within this system, patients are instructed to lean their trunk forward, i.e. to generate motion of the trunk which is similar to the motion of a healthy subject.

Our goal in the present study is to show that such a motion is may reduce the consumed energy and applied shoulder forces using dynamic motion optimization. Obtained results have been experimentally tested.

\section{Method}

\section{$2.1 \quad$ Data collection}

Experimental data were collected from one complete paraplegic patient with lesion at T7 level. Approval from local ethical committee was obtained to run those tests. The kinematics data were acquired by a 4-camera VICON ${ }^{\mathrm{TM}}$ optical system recording position of 16 passive markers at $100 \mathrm{~Hz}$ sample rate. Six degrees of freedom force sensors were mounted on handles fixed on parallel bars in order to record arm efforts. Sampling frequency was $100 \mathrm{~Hz}$. At the beginning, patient was sitting on a chair with arms on

This is an Open Access article distributed under the terms of the Creative Commons Attribution-Noncommercial License 3.0, which permits unrestricted use, distribution, and reproduction in any noncommercial medium, provided the original work is properly cited. 
the handles. The position of the handles was adjusted according to the patient's preference. Patient was instructed to bend the trunk forward prior standing up. Experimenter manually triggered stimulator. The stimulation frequency and pulse width were respectively $30 \mathrm{~Hz}$ and $300 \mu \mathrm{s}$. The stimulation current amplitude was adapted in order to ensure joint locking. The stimulated muscles were quadriceps vastus medialis, hamstring biceps hemoris, gluteus maximus, and tibialis anterior.

\section{2}

\section{Biomechanical Model}

LifeMOD $^{\mathrm{TM}}$ software was used to model human body dynamics [5]. The model consisted of four segments: foot, shank, tight and HAT (head, arms, and trunk) with 3 DOF (ankle, knee and hip). Each joint is modeled as a hinge joint. The connection between foot and support is fixed, i.e. there is no motion between them. Mechanical parameters of the biomechanical model are obtained from LifeMOD's anthropometric database. To take into account the interaction between the human body and the chair to detect the sit off instant, impact force algorithm has been used. In order to simulate upper body standing up activity, vertical (VSF) and horizontal (HSF) force on the shoulders were applied as a step function (Fig.1.). MOCAP data and data from force sensor mounted on handles were used to get trajectories of ankle, knee and hip joint and forces acting on the shoulders.

\section{$2.3 \quad$ Optimization process}

Our objective is to define the optimal strategy for voluntary trunk movement, therefore, the optimization algorithm computes optimal trajectories of hip joint with respect to the trajectories of the knee and ankle measured during the experiment. The trajectories obtained from the optimization process are described through cubic B-spline [6] with 5 control points. The initial and final control point values correspond to sitting and standing position respectively. Optimization process calculates the values of three intermediate control points, dividing the trajectory in 4 equal parts.

In order to minimize lower limbs efforts during sit to stand transition, the chosen cost function is the sum of the squared joint torques in ankle, knee and hip (equation 1), with constrains on the trunk angle (equation 2):

$$
C=\sum_{t o}^{t f}\left(\tau_{a}{ }^{2}+\tau_{k}{ }^{2}+\tau_{h}{ }^{2}\right) \cdot T e
$$

In (1) $\tau_{a}, \tau_{k}$, and $\tau_{h}$ refer to ankle, knee and hip torques respectively, $t_{0}$ is sit off moment, $t f$ is the final time and $T e$ represents the sample time.

$\Theta \min \leq \Theta(t) \leq \Theta \max$

$\Theta \min =-100^{\circ}, \Theta \max =10^{\circ}$

Where $\Theta(t)$ is the trunk angle.

Specific values of the forces acting on the shoulders were chosen to simulate 2 different sit-to-stand conditions. In the first optimization process, the force acting on the shoulder was set to be equal to the one obtained from experimental data. We are interested in decreasing arm forces during sit to stand motion, therefore in the second optimization, VSF and HSF were chosen to be null, i.e. without influence of the arm support.

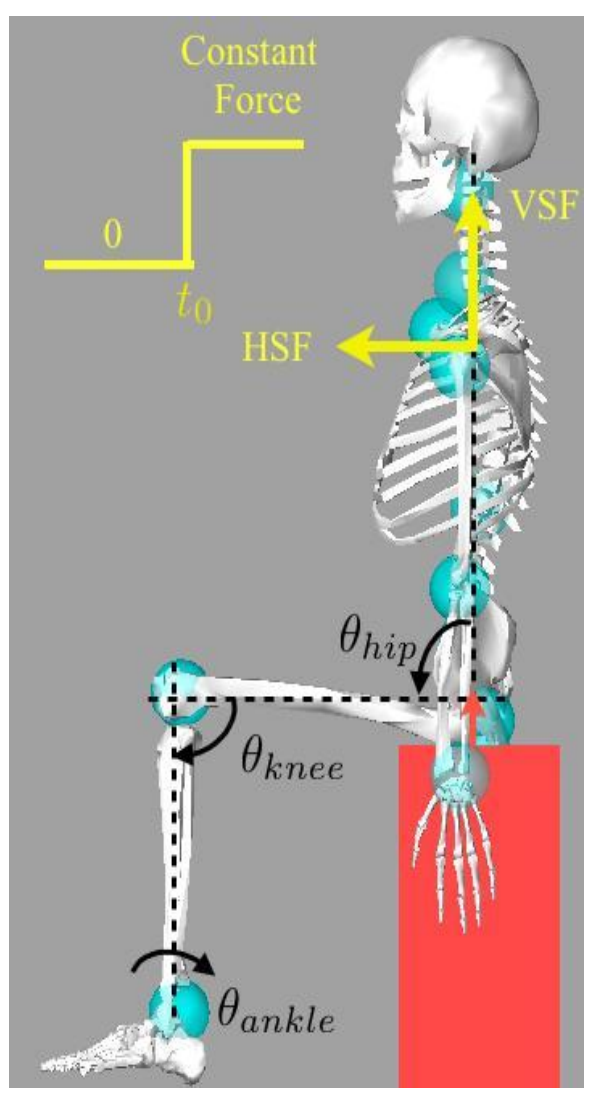

Fig. 1. Model, VSF and HSF are vertical and horizontal component of the shoulder force respectively, $\mathrm{t}_{0}$ is sit off moment when constant force on the shoulder was applied. Oankle, Oknee, and Ohip are ankle angle, knee angle and hip angle respectively. The initial joint position vector was $\Theta=\left[0^{\circ}, 90^{\circ},-80^{\circ}\right]$. 


\section{4}

\section{Experimental validation}

Experiments have been performed with one T6 paraplegic patient in order to validate optimization results. At the beginning of the experiment subject was sitting on the chair with arms rested on the handles. Subject performed rising motion using our system which should automatically trigger leg stimulation in optimal moment with respect to the trunk acceleration in order to decrease arm participation during the motion. Subject bent his trunk forward before sit off phase. The trunk acceleration was acquired by one-axis wireless accelerometer positioned between shoulder blades of the subject. The wireless system is described in [7]. Six degrees of freedom force sensors were mounted on handles on parallel bars in order to record arm efforts. Sampling frequency of accelerometer and force sensors was $100 \mathrm{~Hz}$. The stimulation parameters were the same as reported in section 2.1. To ensure smoother muscle contraction, ramping of stimulation train was used with ramp duration of $300 \mathrm{~ms}$. Stimulation muscles were quadriceps vastus medialis, hamstring biceps hemoris and tibialis anterior.

\section{Results and Discussion}

In figure 2 trajectories for ankle, knee and hip measured during data collection experiment are presented. The amplitude of the reaction forces acting on the left shoulder was $22 \%$ and $3.5 \%$ of the patient's body weight (BW) for vertical and horizontal component respectively. Results of the optimization algorithm are presented on Fig 3. In Fig. 3a) amplitudes of applied vertical and horizontal shoulder forces was 22 and 3,5 percentage of patient's BW respectively, i.e. had the same numerical value as the shoulder force calculated from experimental data. The results of optimization process suggested that in this case forces provided by upper extremities and stimulated lower limbs are enough to lift body upward and inertia of trunk is neither necessary nor optimal in terms of joint torques.

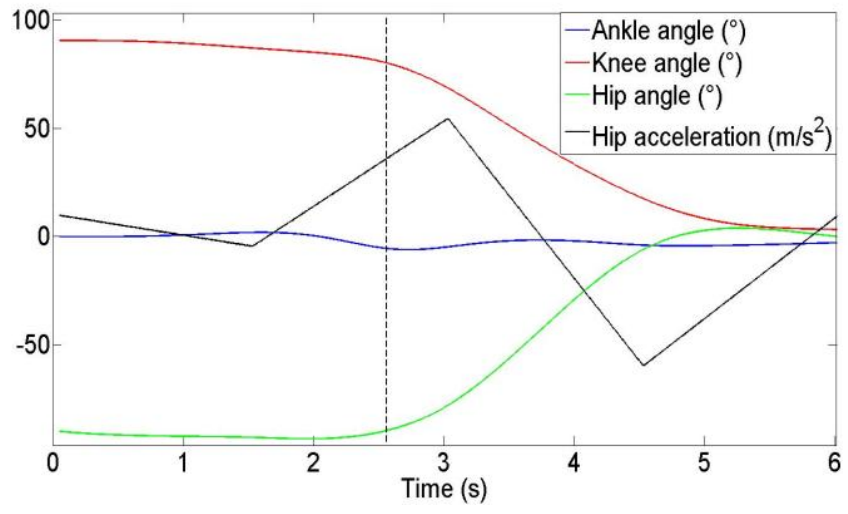

Fig. 3.a) Optimization result with vertical shoulder force of $22 \% \mathrm{BW}$ and horizontal shoulder force of $3,5 \% \mathrm{BW}$. Blue line represents ankle angle, red line is knee angle, and green line is hip angle. The dashed bar marks the beginning of sit off phase.

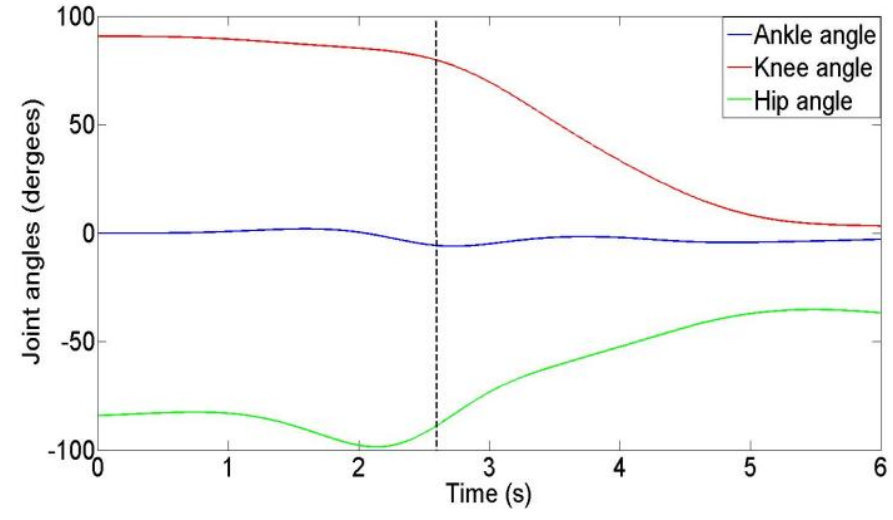

Fig. 2 Lower limb trajectories measured during the data collection experiment. Blue line is ankle angle, red line is knee angle and green line is hip angle. The dashed bar marks the beginning of sit off phase.

In Fig. 3b) optimization results without arm support are presented. In this case, the results suggest that the patient should use a strategy similar to one of healthy person: prior standing up, he should bend his body forward in order to use linear momentum of their trunk which is helpful during the lift of rising phase [4]. Knee extension should start around $600 \mathrm{~ms}$ before maximum of the trunk acceleration, and to finish in approximately same time as trunk motion. Fig.4. shows mean and maximal values of the sum of left and right hand forces measured during the experimental validation. Stimulation time in seconds with respect to the maximal trunk acceleration is presented on the $\mathrm{x}-$ axis. Negative values indicate that stimulator was triggered before maximum of trunk acceleration. In the same way, positive values mean that stimulation started after. From the Fig.4. it seems that there is an influence of the timing of stimulation application and upper limb support forces. As expected, the lowest mean and maximal value of hand forces were achieved for trials when stimulation started around $0.3 \mathrm{~s}$ before maximal trunk acceleration occurrence (ramp duration was $300 \mathrm{~ms})$.

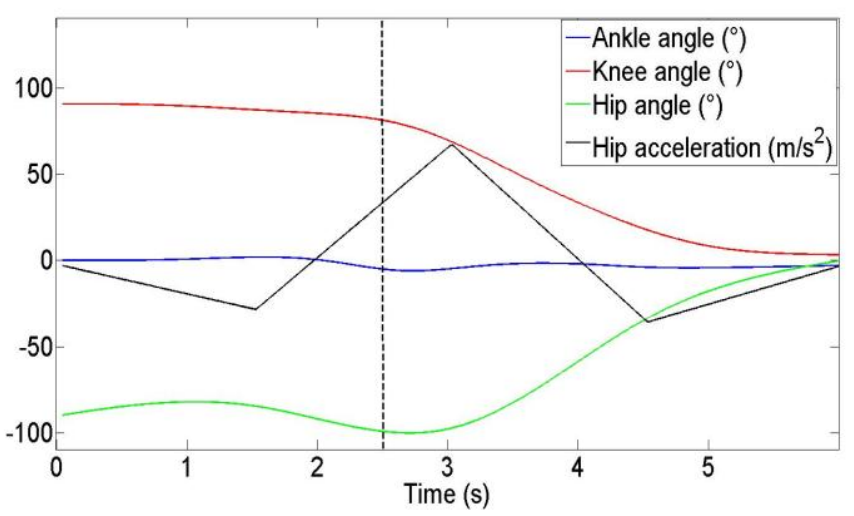

Fig. 3.b) Optimization result without applied shoulder force. Blue line represents ankle angle, red line is knee angle, and green line is hip angle. The dashed bar marks the beginning of sit off phase. 


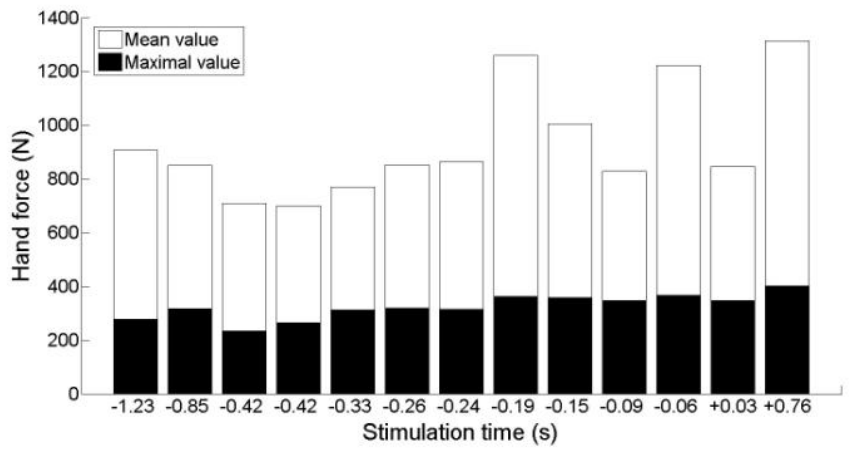

Fig. 4. Mean and maximal value of sum of the left and right hand forces measured during experimental validation.

\section{Conclusion}

From the results presented here following conclusions can be made. First, among different strategies which paraplegic patients practice to standup [9], the most optimal would be similar with one of the healthy people. In other words, in order to reduce lifting forces provided from upper extremities, patient should bend his body forward and stimulation should be triggered before the maximum trunk acceleration in order to use linear momentum of the trunk in sit off phase. Second, it is possible to find theoretical optimal time instant with respect to the trunk motion when simulation should start, which would minimize arm forces and sum of the ankle, knee, and hip torques during sit-to-stand motion. Third, our closed-loop controller appears to be a good solution to trigger stimulation at the appropriate timing [8]. Using the described system enables us to start the stimulation at optimal moment during sit-to-stand movement, something not feasible with manual triggering.

Further study will involve more paraplegic subjects in order to evaluate the closed-loop system and to generalize the conclusions of this paper.

\section{References}

[1] PO Riley, ML Schenkman, RW Mann, WA Hodge, Mechanics of a constrained chair-rise, J Biomech., vol. 24, pp. 77-85, 1991

[2] C Azevedo, R Heliot, Rehabilitation of functional posture and walking: Coordination of healthy and impaired limbs, Journal of automatic control, vol. 15, pp $11-15,2005$

[3] J Jovic, C Azevedo Coste, P Fraisse, C Fattal, Optimizing FES-assisted sit to stand transfer initiation in paraplegic individuals using trunk movement information, The XVIII Congress of the International Society of Electrophysiology and Kinesiology, Aalborg, Denmark, 2010.

[4] R Heliot, C Azevedo, B Espiau, D Dominique, Early detection of postural modification and motion monitoring using micro attitude sensor, AMAM Conference, Ilmenau, Germany, 2005

[5] http://www.lifemodeler.com

[6] C de Boor, Practical guide to splines, Springer-Verlag, New York, 1978.

[7] C Azevedo Coste, R Hélio, R Pissard-Gibollet, P Dussaud, D Andreu, F Jérôme, I Laffont, MASEA : Marche Assistée par Stimulation Électrique Adaptative, Sciences et Tech. pour le Handicap, vol 4, pp 109-130, 2010.

[8] J Jovic, C Azevedo Coste, P Fraisse, C Fattal, Upper and lower body coordination in FES-assisted sit-to-stand transfers in paraplegic subjects - A case study, IEEE Trans. Neur. Syst. Rehabil. Eng., submitted.

[9] R Kamnik, T Bajd, A Kralj, Functional electrical stimulation and arm supported sit-to-stand transfer after paraplegia: a study of kinetic parameters, Artificial Organs, vol. 23, pp. 413-417, 1999.

[10] J Jovic, V Bonnet, P Fraisse, C Fattal, C Azevedo Coste, Improving valid and deficient body segment coordination to improve FES assisted sit to stand in paraplegic subjects, ICORR, 2011.

[11] R Kaminik, J Qing Shi, R Murray-Smith, T Bajd, Nonlinear modeling of FES-supported standing-up in paraplegia for selection of feedback sensors, IEEE Trans. Neur. Syst. Rehabil. Eng, vol 13, pp. 40-52, 2005. 